03,13

\title{
Дефекты межслоевой поверхности и термоэлектрические свойства в слоистых пленках топологических изоляторов $n-\mathrm{Bi}_{2} \mathrm{Te}_{2.7} \mathrm{Se}_{0.15} \mathrm{~S}_{0.15}$
}

\author{
(С) Л.Н. Лукьянова, О.А. Усов, М.П. Волков, И.В. Макаренко, В.А. Русаков \\ Физико-технический институт им. А.Ф. Иофрфе РАН, \\ Санкт-Петербург, Россия \\ E-mail: lidia.lukyanova@mail.ioffe.ru
}

Поступила в Редакцию 13 мая 2021 г.

В окончательной редакции 13 мая 2021 г.

Принята к публикации 13 мая 2021 г.

В слоистых пленках топологических изоляторов $n-\mathrm{Bi}_{2} \mathrm{Te}_{2.7} \mathrm{Se}_{0.15} \mathrm{~S}_{0.15}$, оптимизированных для температур ниже комнатной, исследованы морфология межслоевой поверхности (0001) и термоэлектрические свойства. На профилях поверхности (0001) идентифицированы нейтральные примесные дефекты, возникающие при замещениях атомов Те на атомы $\mathrm{Se}$ и $\mathrm{S}$ и донорные антиструктурные дефекты теллура на местах висмута, которые влияют на термоэлектрические свойства.

Среднее значение термоэлектрической эффективности в пленках $n$ - $\mathrm{Bi}_{2} \mathrm{Te}_{2.7} \mathrm{Se}_{0.15} \mathrm{~S}_{0.15}$ возрастает до $\langle Z\rangle \approx 3.0 \cdot 10^{-3} \mathrm{~K}^{-1}$ в интервале $80-215 \mathrm{~K}$, в то время как в объемном твердом растворе $\langle Z\rangle \approx 2.0 \cdot 10^{-3} \mathrm{~K}^{-1}$. Рост термоэлектрической эффективности в пленках связан с усилением энергетической

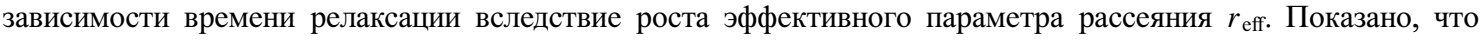
в пленках коэффициент Зеебека, эффективная масса плотности состояний $m / m_{0}$ и параметр материала, пропорциональный фактору мощности, возрастают, а решеточная $\kappa_{L}$ и электронная теплопроводность $\kappa_{e}$ уменьшаются, что определяет повышение термоэлектрической эффективности.

Ключевые слова: твердые растворы на основе теллурида висмута, пленки, топологический изолятор, дефекты, термоэлектрические свойства.

DOI: 10.21883/FTT.2021.10.51394.111

\section{1. Введение}

Термоэлектрические материалы на основе слоистых халькогенидов висмута и сурьмы с оптимальными свойствами для интервала температур $100-500 \mathrm{~K}$ в зависимости от состава и концентрации носителей заряда являются не только эффективными термоэлектриками [1], но и перспективными трехмерными топологическими изоляторами (ТИ) с аномальными электронными свойствами поверхностных состояний фермионов Дирака [2,3]. Для фермионов в ТИ характерна линейная дисперсия и жесткая связь между спином и импульсом в силу симметрии относительно инверсии времени, что препятствует обратному рассеянию фермионов на немагнитных примесях и дефектах, и способствует увеличению подвижности носителей заряда [4].

В настоящее время ТИ являются перспективными материалами для применений в различных областях физики. В фотонике $\mathrm{Bi}_{2} \mathrm{Te}_{3}$ применяется для пассивной синхронизации сверхскоростных оптоволоконных лазеров в системах оптической связи [5,6]. В спинтронике операция преобразования между спином и зарядом при комнатной температуре реализуется в ТИ на несколько порядков эффективнее, чем тяжелых металлах $[7,8]$. В термоэлектричестве и электронике рассматривается возможность использования эффекта свертекучести экситонов $[9,10]$.
Сильное спин-орбитальное взаимодействие и нетривиальная зонная структура фермионов Дирака в топологических изоляторах являются причиной усиления энергетической зависимости спектрального распределения длин свободного пробега электронов в исследуемых материалах и, соответственно, увеличения коэффициента Зеебека за счет фильтрации носителей заряда $[11,12]$. Аналогичное увеличение коэффициента Зеебека в топологических термоэлектриках на основе $\mathrm{Bi}_{2} \mathrm{Te}_{3}$ [13] согласуется с оценками, выполненными в рамках теории теплового квантового поля при появлении электроннодырочных пар Швингера на горячей стороне термоэлектрика вдоль градиента температуры.

Значительный интерес представляют теоретические $[14,15]$ и экспериментальные $[16,17]$ исследования эффекта сверхтекучести бозе-энштейновского конденсата межслоевых [15] экситонов, известных также как топологические [18] или двухслойные [9] экситоны, которые возникают благодаря сильному кулоновскому взаимодействию электронно-дырочных токов в пространственно разделенных проводящих слоях ТИ. В тонкопленочных двухслойных экситонных структурах на основе $n$ - и $p$-типа $\mathrm{Bi}_{2} \mathrm{Te}_{3}$, разделенных изолирующим слоем, за счет кулоновского взаимодействия электроннодырочных пар на границе полупроводника и изолятора возникают межслоевые экситоны при оптимальных толщинах пленок и изолирующих слоев. По оценкам $[10,19]$ сопротивление, связанное с эффектом кулоновского 
увлечения, становится существенно меньше, чем сопротивление каждой пленки, подвижность носителей заряда увеличивается на порядок, что позволяет использовать двухслойные экситонные структуры для увеличения термоэлектрической эффективности.

Исследования термоэлектрических свойств при комнатной температуре в области высоких давлений в субмикронных монокристаллических слоистых пленках твердых растворов $\mathrm{Bi}_{2} \mathrm{Te}_{3}$ при замещениях атомов в подрешетках Ві и Те показали, что параметр мощности возрастает в $2.5-3$ раза за счет роста подвижности [20,21]. Максимум параметра мощности наблюдался в области давлений 3-4 GPa, где происходит изоструктурный топологический фазовый переход [22,23] в результате усиления гибридизации волновых функций электронов и формирования инверсионных зон в области уровня Ферми. Таким образом, имеющиеся теоретические и экспериментальные результаты, учитывающие влияние топологических поверхностных состояний на термоэлектрические свойства в материалах на основе $\mathrm{Bi}_{2} \mathrm{Te}_{3}$, подтверждают перспективность исследований в этой области.

В настоящей работе рассматриваются термоэлектрические свойства в слоистых пленках топологического изолятора $n$ - $\mathrm{Bi}_{2} \mathrm{Te}_{2.7} \mathrm{Se}_{0.15} \mathrm{~S}_{0.15}$, оптимизированного для области низких температур с высоким коэффициентом Зеебека. Морфология межслоевой поверхности (0001), на которой проводились измерения термоэлектрических свойств, была исследована методом сканирующей туннельной микроскопии (СТМ) с целью идентификации дефектов, которые оказывают влияние на термоэлектрические свойства. Выбор пленок указанного состава связан с результатами исследований микрорамановского рассеяния [24] и дифференциальной туннельной проводимости методом сканирующей туннельной спектроскопии (СТC) [25], которые показали, что влияние поверхностных состояний фермионов Дирака возрастает в подобных составах твердых растворов с высокими значениями коэффициента Зеебека.

\section{2. Морфология межслоевой поверхности: анализ изображений и систематизация дефектов}

Исследование морфологии межслоевой поверхности (0001) в слоистых монокристаллах $n$ - $\mathrm{Bi}_{2} \mathrm{Te}_{2.7} \mathrm{Se}_{0.15} \mathrm{~S}_{0.15}$ методом СТМ с атомным разрешением позволяет получить детальную информацию о дефектах и провести их идентификацию, что представляет значительный интерес, поскольку влияние дефектов на процессы рассеяния во многом определяет термоэлектрические свойства.

Морфологию поверхности (0001) в монокристаллах твердого раствора $n$ - $\mathrm{Bi}_{2} \mathrm{Te}_{2.7} \mathrm{Se}_{0.15} \mathrm{~S}_{0.15}$ исследовали с помощью сканирующего туннельного микроскопа GPI-300 (ИОФ РАН, Москва) в комплекте с высоковакуумным

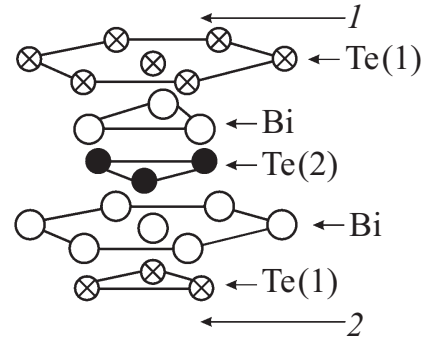

Pис. 1. Пятислойный пакет $\mathrm{Bi}_{2} \mathrm{Te}_{3}$. Стрелками 1,2 показаны щели Ван-дер-Ваальса.

модулем, разработанным в ФТИ им. А.Ф. Иоффе, СПб. Измерения методом СТМ были выполнены при комнатной температуре в высоковакуумной камере при давлении $2.4 \cdot 10^{-7} \mathrm{~Pa}$ в режиме постоянного тока с разрешением $0.05 \AA$.

Образцы твердых растворов $n-\mathrm{Bi}_{2} \mathrm{Te}_{2.7} \mathrm{Se}_{0.15} \mathrm{~S}_{0.15}$ для исследований морфологии межслоевой поверхности Ван-дер-Ваальса (0001) вырезали из монокристаллических блоков, ориентированных по оси роста объемных слитков, полученных методом направленной кристаллизации. Ось роста, перпендикулярная кристаллографической оси $c_{3}$ кристалла, направлена вдоль межслоевых плоскостей (0001). Твердые растворы $n-\mathrm{Bi}_{2} \mathrm{Te}_{2.7} \mathrm{Se}_{0.15} \mathrm{~S}_{0.15}$, как и базовое бинарное соединение $\mathrm{Bi}_{2} \mathrm{Te}_{3}$, кристаллизуются в ромбоэдрической структуре с пространственной группой $R \overline{3} m\left(D_{3 \mathrm{~d}}^{5}\right)$. Кристаллическая структура рассматриваемых твердых растворов представляет собой анизотропные слои, которые состоят из пятислойных пакетов или квинтетов, разделенных щелями Ван-дер-Ваальса (рис. 1). Атомные слои в квинтете теллурида висмута чередуются в последовательности $(-\mathrm{Te}(1)-\mathrm{Bi}-\mathrm{Te}(2)-\mathrm{Bi}-\mathrm{Te}(1)-)$.

Атомы $\mathrm{Te}(1)$ и $\mathrm{Te}(2)$ имеют различное ближайшее окружение. Атомы Те(2) имеют октаэдрическое окружение атомами $\mathrm{Bi}$, а атомы $\mathrm{Te}(1)$ в качестве ближайших соседей имеют 3 атома Ві и 3 атома Те(1). В твердом растворе $n$ - $\mathrm{Bi}_{2} \mathrm{Te}_{2.7} \mathrm{Se}_{0.15} \mathrm{~S}_{0.15}$ атомы $\mathrm{Se}$ и $\mathrm{S}$ замещают атомы Те(2) [26]. Химические связи между атомами в слоях квинтета ковалентные с небольшой долей ионности, а квинтеты связаны слабыми силами Ван-дер-Ваальса, что обеспечивает расслоение кристаллов по межслоевой поверхности (0001). Изображения морфологии поверхности (0001) в твердом растворе $n-\mathrm{Bi}_{2} \mathrm{Te}_{2.7} \mathrm{Se}_{0.15} \mathrm{~S}_{0.15}$ соответствуют кристаллической гексагональной плотноупакованной структуре (рис. 2), что согласуется с СТМ исследованиями материалов на основе теллурида висмута различного состава $[11,27]$.

Флюктуации интенсивности, наблюдаемые на морфологии поверхности (0001) в твердом растворе $n$ - $\mathrm{Bi}_{2} \mathrm{Te}_{2.7} \mathrm{Se}_{0.15} \mathrm{~S}_{0.15}$, связаны с искажениями поверхностных электронных состояний при неравномерном распределении кулоновского потенциала $[27,28]$ в результате замещения атомов в подрешетке Те и образования 

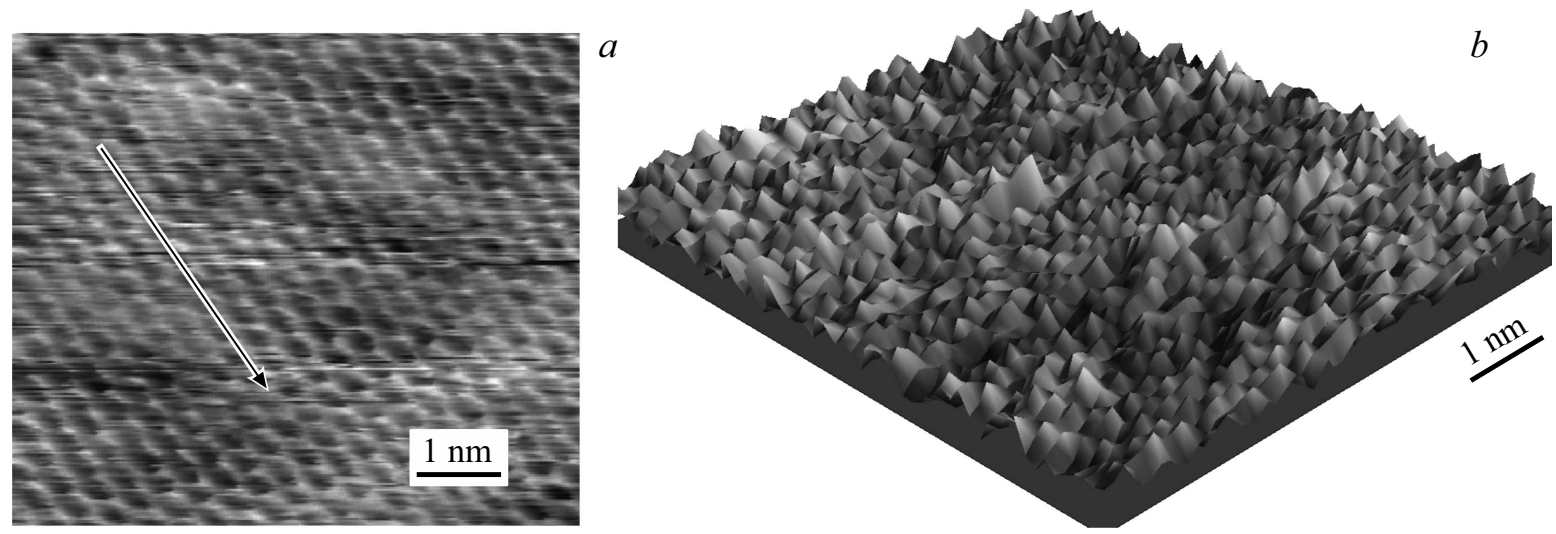

Рис. 2. Двухмерное $(a)$ и соответствующее трехмерное $(b)$ изображения морфологии межслоевой поверхности $(0001)$ в твердом растворе $n-\mathrm{Bi}_{2} \mathrm{Te}_{2.7} \mathrm{Se}_{0.15} \mathrm{~S}_{0.15}$.

собственных дефектов, которые формируются в процессе роста твердых растворов (рис. 2). Оценка искажений поверхностных электронных состояний проводилась с помощью гистограмм рельефа поверхности (0001) (рис. $2, a, b$ ), из которых определяли средние значения $H_{M}$ и среднеквадратичные отклонения $H_{S}$ искажений по высоте в распределении атомов на поверхности. Средние величины $H_{M}$ и $H_{S}$, рассчитанные по семи изображениям морфологии поверхности (0001) в $n-\mathrm{Bi}_{2} \mathrm{Te}_{2.7} \mathrm{Se}_{0.15} \mathrm{~S}_{0.15}$, составляют 0.078 и $0.019 \mathrm{~nm}$ соответственно.

На рис. 3 приведены профили поверхности (0001), на которых в виде выступов и провалов видны искажения в распределении высот между соседними атомами, вызванные влиянием примесных и собственных дефектов.

В твердом растворе $n$ - $\mathrm{Bi}_{2} \mathrm{Te}_{2.7} \mathrm{Se}_{0.15} \mathrm{~S}_{0.15}$ наиболее вероятно образование нейтральных примесных дефектов

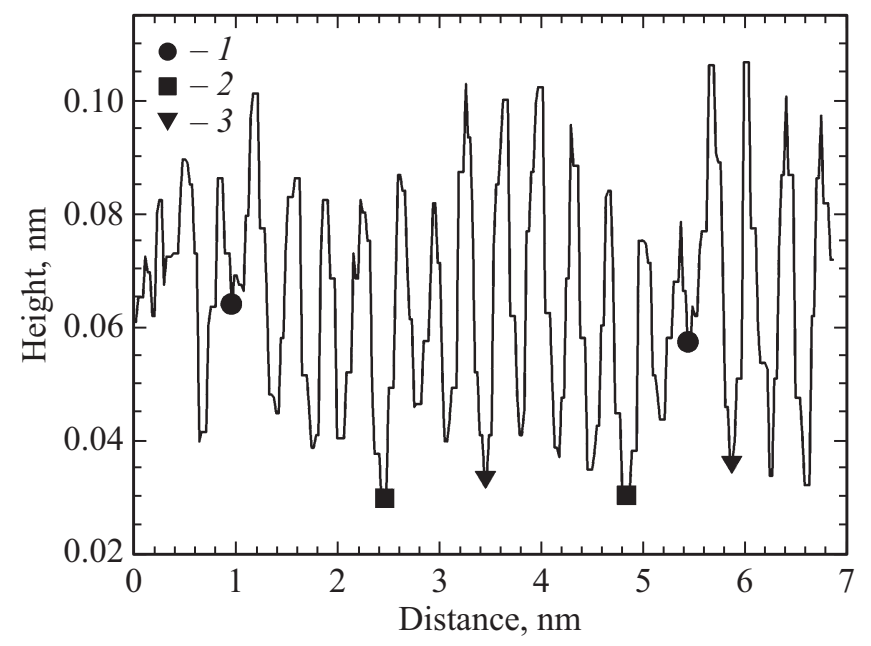

Рис. 3. Профиль поверхности (0001) вдоль линии, показанной на рис. 2, в твердом растворе $n-\mathrm{Bi}_{2} \mathrm{Te}_{2.7} \mathrm{Se}_{0.15} \mathrm{~S}_{0.15}$. Поверхностные дефекты: 1 - антиструктурные дефекты Те $\mathrm{Bi}_{\text {, }}$ 2,3 - примесные дефекты замещения $-\mathrm{Se} \rightarrow \mathrm{Te}$ и $\mathrm{S} \rightarrow \mathrm{Te}$ соответственно.
Ковалентные атомные радиусы $\mathrm{Bi}, \mathrm{Te}, \mathrm{Se}$, и $\mathrm{S}$, использованные для идентификации дефектов

\begin{tabular}{c|c|c|c|c}
\hline \multicolumn{5}{c}{ Ковалентные атомные радиусы, $\mathrm{nm}$} \\
\hline Ссылки & $\mathrm{Bi}$ & $\mathrm{Te}$ & $\mathrm{Se}$ & $\mathrm{S}$ \\
\hline$[33]$ & 0.16 & 0.14 & 0.115 & 0.100 \\
{$[31]$} & 0.15 & 0.142 & 0.119 & \\
{$[28]$} & 0.146 & 0.136 & 0.116 & 0.102
\end{tabular}

замещения $\mathrm{Se}-\mathrm{Te}$ и $\mathrm{S}-\mathrm{Te}$ и донорных антиструктурных дефектов теллура на местах висмута $\mathrm{Te}_{\mathrm{Bi}}$, т.е. $\mathrm{Te}(1)$ занимает места Ві во втором слое пятислойного пакета (рис. 1). Энергия образования рассматриваемых дефектов ниже, чем энергия, необходимая для появления вакансий и адатомов, которые также могут наблюдаться в материалах на основе $\mathrm{Bi}_{2} \mathrm{Te}_{3}[25,26,29,30]$. Однако, в составах $n$-типа $\mathrm{Bi}_{2} \mathrm{Te}_{2} \mathrm{Se}[31]$, как и в настоящей работе, были также обнаружены дефекты замещения $\mathrm{Se}-\mathrm{Te}$ и антиструктурные дефекты $\mathrm{Te}_{\mathrm{Bi}}$. В [32] на основе численных расчетов методом функционала плотности было показано, что пары антиструктурных дефектов имеют тенденцию располагаться на ближайших друг от друга расстояниях около $0.5-1.5 \mathrm{~nm}$, что оказывает влияние на структуру и термоэлектрические свойства материала.

Как показано на рис. 3, на профиле поверхности (0001) в $n-\mathrm{Bi}_{2} \mathrm{Te}_{2.7} \mathrm{Se}_{0.15} \mathrm{~S}_{0.15}$ антиструктурные дефек-

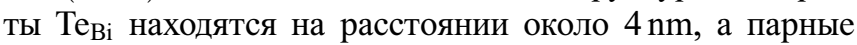
антиструктурные дефекты не были обнаружены.

Обычно идентификация дефектов проводится путем сравнения изображений дефектов, полученных СТМ на бинарных материалах при низких температурах, с расчетными изображениями этих дефектов $[26,29]$. При измерениях морфологии поверхности (0001) при комнатной температуре, характер дефектов можно оценить по величинам провалов на профилях поверхности, используя значения атомных радиусов $\mathrm{Bi}, \mathrm{Te}, \mathrm{Se}$ и $\mathrm{S}$, приведенные в таблице. 


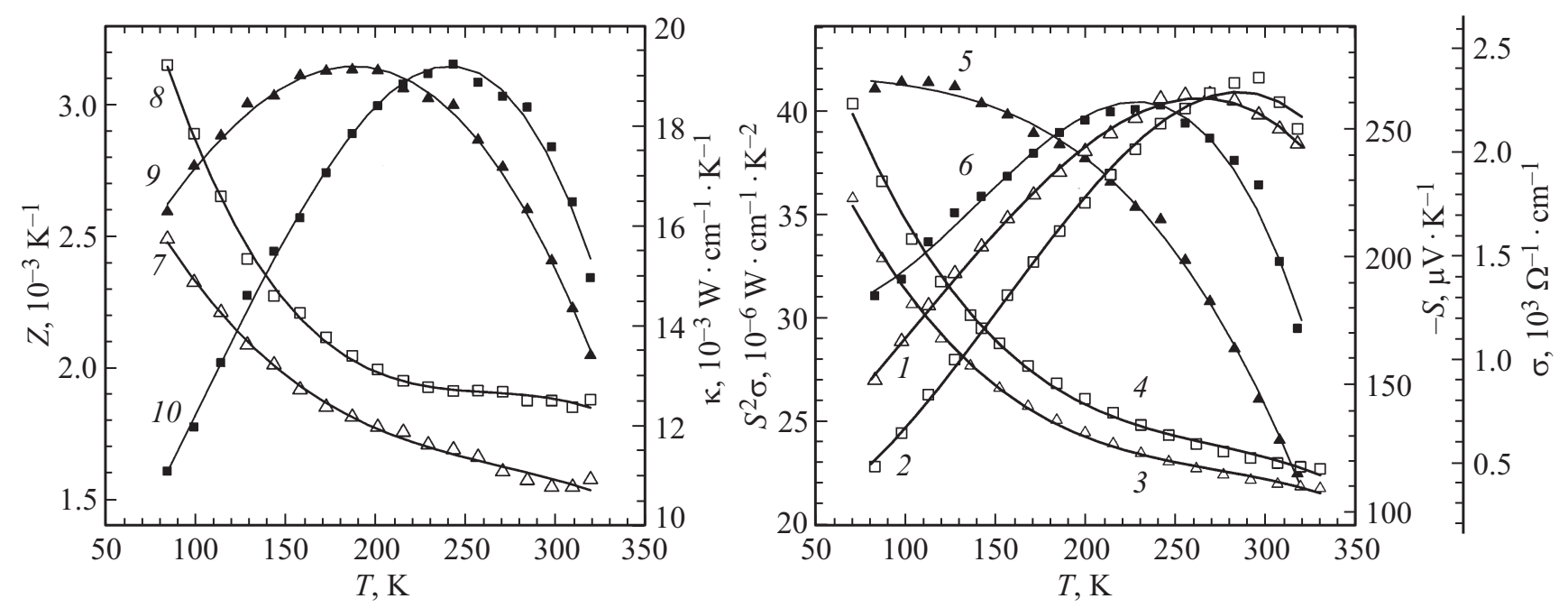

Рис. 4. Температурные зависимости коэффициента Зеебека $S(1,2)$, электропроводности $\sigma(3,4)$, фактора мощности $S^{2} \sigma(5,6)$, теплопроводности $\kappa(7,8)$ и термоэлектрической эффективности $Z(9,10)$ в слоистой пленке $(1,3,5,7,9)$ и объемном твердом растворе $(2,4,6,8,10) n-\mathrm{Bi}_{2} \mathrm{Te}_{2.7} \mathrm{Se}_{0.15} \mathrm{~S}_{0.15}$.

В первом приближении дефектам замещения и антиструктурным дефектам соответствуют величины провалов на профилях поверхности (0001), равные разности диаметров атомов Те, Se и Te, S. На рис. 2 отмечены величины провалов на профилях поверхности (0001) в $n-\mathrm{Bi}_{2} \mathrm{Te}_{2.7} \mathrm{Se}_{0.15} \mathrm{~S}_{0.15}$ для примесных дефектов замещения $\mathrm{Se}-\mathrm{Te}$ и $\mathrm{S}-\mathrm{Te}$, которые составляют около 0.05 и $0.08 \mathrm{~nm}$, соответственно, а для антиструктур-

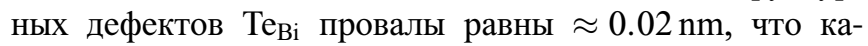
чественно согласуется с работами [28,33], (таблица). Величины провалов на профилях поверхности (0001) в $n-\mathrm{Bi}_{2} \mathrm{Te}_{2.7} \mathrm{Se}_{0.15} \mathrm{~S}_{0.15}$ для примесных дефектов (рис. 3) согласуются с результатами для твердого раствора $\mathrm{Bi}_{2} \mathrm{Te}_{2} \mathrm{Se}[31]$, в которых величины провалов рассчитывали в структурной модели для двух верхних слоев пятислойного пакета (рис. 1).

\section{3. Термоэлектрические свойства}

В слоистых пленках твердого раствора $n-\mathrm{Bi}_{2} \mathrm{Te}_{2.7} \mathrm{Se}_{0.15} \mathrm{~S}_{0.15}$, полученных последовательным механическим расслоением монокристаллических блоков, в которых были проведены исследования морфологии поверхности (0001) (рис. 2,3), измерены термоэлектрические свойства в зависимости от температуры на установке Physical Property Measurement System (PPMS), Thermal Transport Option (рис. 4). Многократное механическое расслоение с помощью липкой ленты позволяет получать пленки твердых растворов на основе теллурида висмута от субмикронной толщины до 100-120 nm.

В пленке $n$ - $\mathrm{Bi}_{2} \mathrm{Te}_{2.7} \mathrm{Se}_{0.15} \mathrm{~S}_{0.15}$ коэффициент Зеебека возрастает по сравнению с объемным термоэлектриком в области температур 80-270 K, а угловые коэффициенты температурных зависимостей $d \ln S / d \ln T$, определенные на участках, близким к линейным, снижаются (рис. 4, кривые 1,2), т.е. зависимости $S(T)$ в пленке ослабевают. Величины $d \ln S / d \ln T$ в пленке равны 0.55 и 0.48 на линейных участках в интервалах температур $T=83-142 \mathrm{~K}$ и $142-214 \mathrm{~K}$, соответственно. В объемном образце аналогичные величины составляют 0.63 $(T=83-98 \mathrm{~K})$ и $0.735(T=98-214 \mathrm{~K})$.

Наибольшее снижение угловых коэффициентов в пленках наблюдается при температурах ниже и вблизи температуры Дебая $T_{D}$, что объясняется преимущественным влиянием рассеяния на точечных дефектах: собственных донорных антиструктурных дефектах и нейтральных примесных дефектах, возникающих при замещении атомов теллура на селен и серу при образовании твердого раствора (рис. 3). Такой характер зависимости $S(T)$ в пленке указывает на особенности механизма рассеяния носителей заряда вследствие влияния поверхностных состояний фермионов Дирака, и обеспечивает рост фактора мощности, компенсируя незначительное снижение электропроводности $\sigma$ по сравнению с объемным образцом (рис. 4, кривые 3-6). Полученное снижение $\sigma$ в пленке по сравнению объемным образцом (рис. 4, кривые 3,4), определяется соотношением между объемной и поверхностной проводимостью, которое зависит от наличия дефектов в объеме топологического изолятора при частичной компенсации вкладов в проводимость акцепторных и донорных собственных дефектов [34-36].

Рост фактора мощности и снижение теплопроводности обеспечивают повышение термоэлектрической эффективности в пленке $n-\mathrm{Bi}_{2} \mathrm{Te}_{2.7} \mathrm{Se}_{0.15} \mathrm{~S}_{0.15}$ при температурах ниже $215 \mathrm{~K}$ по сравнению с объемным термоэлектриком (рис. 4, кривые 5-8). В исследованной слоистой пленке при $T=80 \mathrm{~K}$ величина $Z=2.6 \cdot 10^{-3} \mathrm{~K}^{-1}$, а в объемном материале, оптимизированном для области низких температур, эффективность $Z=1.6 \cdot 10^{-3} \mathrm{~K}^{-1}$, в 
то время как в стандартных термоэлектриках $Z$ обычно составляет $(0.3-0.5) \cdot 10^{-3} \mathrm{~K}^{-1}$. В интервале $80-215 \mathrm{~K}$ средние значения термоэлектрической эффективности $\langle Z\rangle=3.0 \cdot 10^{-3} \mathrm{~K}^{-1}$ для пленки и $2.0 \cdot 10^{-3} \mathrm{~K}^{-1}$ для объемного термоэлектрика (рис. 4 , кривые 9, 10).

\section{4. Анализ термоэлектрических свойств с учетом эфффективного параметра рассеяния}

Экспериментальные температурные зависимости термоэлектрических свойств были проанализированы с помощью величин эффективной массы плотности состояний $m / m_{0}$, подвижности носителей заряда $\mu_{0}$, теплопроводности кристаллической решетки $\kappa_{L}$ и электронной теплопроводности $\kappa_{e}$. Величины $m / m_{0}, \mu_{0}, \kappa_{L}$ и $\kappa_{e}$ в зависимости от температуры были определены в модели энергетического спектра с изотропным рассеянием носителей заряда с учетом эффективного параметра рассеяния $r_{\text {eff }}$ в выражении времени релаксации $\tau=\tau_{0} E^{r}$, $\tau_{0}$ - константа, не зависящая от энергии, $r$ - текущее значение $r_{\text {eff }}$ здесь и далее [37].

Эффективный параметр рассеяния $r_{\text {eff }}$ и приведенный уровень Ферми $\eta$ определяются коэффициентом Зеебека $S$ (1) и параметром вырождения $\beta_{\mathrm{d}}(2)$, которые выражаются через функции Ферми $F_{r+n}(\eta)$, где $n=0.5$, $1.5,2.5$ в виде

$$
\begin{gathered}
S=\frac{k}{e}\left[\frac{(r+5 / 2) F_{r+3 / 2}(\eta)}{(r+3 / 2) F_{r+1 / 2}(\eta)}-\eta\right], \\
\beta_{d}(r, \eta)=\frac{(2 r+3 / 2)^{2} F_{2 r+1 / 2}^{2}(\eta)}{(r+3 / 2)(3 r+3 / 2) F_{r+1 / 2}(\eta) F_{3 r+1 / 2}(\eta)} .
\end{gathered}
$$

Величины $r_{\text {eff }}$ и $\eta$ в пленке и объемном твердом растворе $n$ - $\mathrm{Bi}_{2} \mathrm{Te}_{2.7} \mathrm{Se}_{0.15} \mathrm{~S}_{0.15}$ были рассчитаны методом МНК с использованием данных о параметре вырождения $\beta_{\mathrm{d}}$ в соответствии с [37]. Функции Ферми $F_{r+n}(\eta)$ (рис. 5, кривые 1-6) рассчитывали для соответствующих значений $r_{\text {eff }}$ (рис. 5, кривые 7,8 ).

При построении зависимостей $\mathrm{S}\left(r_{\text {eff }}\right)$ (рис. 5, кривые 7,8$)$ использовали величины коэффициента Зеебека, приведенные на рис. 4, кривые 1, 2, для всех температур, при которых проведены измерения. Увеличение $\left|r_{\text {eff }}\right|$ в пленках $n$ - $\mathrm{Bi}_{2} \mathrm{Te}_{2.7} \mathrm{Se}_{0.15} \mathrm{~S}_{0.15}$ определяет более резкую энергетическую зависимость времени релаксации $\tau$ по сравнению с объемным материалом, что объясняется эффектом фильтрации носителей заряда и приводит к росту коэффициента Зеебека в области температур 80-240 K (рис. 4, кривые 1,2) в пленках благодаря более широкому энергетическому спектру электронов в топологических изоляторах $[5,9,13,22]$.

Как и для зависимостей $S(T)$, особенности $m / m_{0}(T)$ определяются влиянием эффекта фильтрации носителей заряда в пленках топологических изоляторов. Эффективная масса $m / m_{0}$ значительно выше в пленке

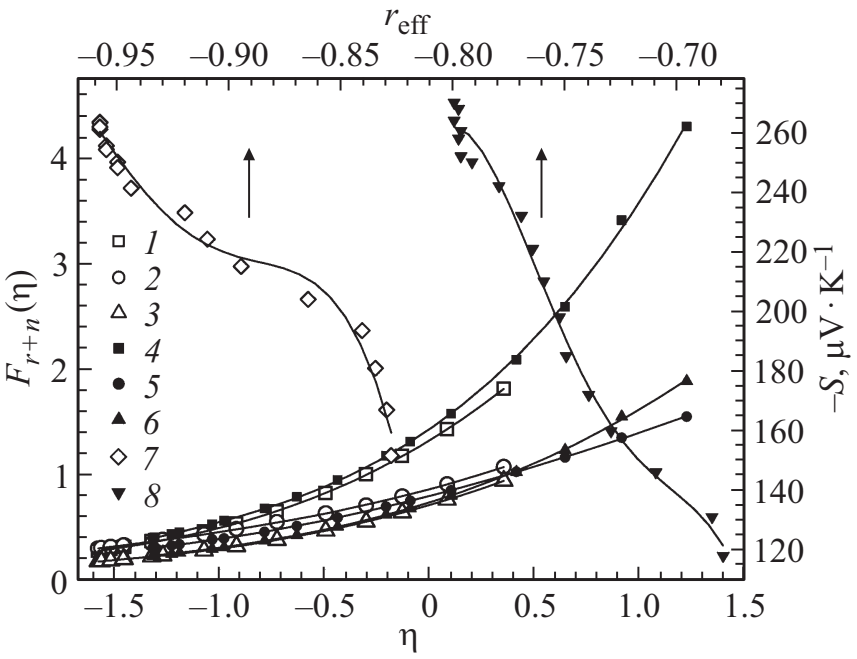

Рис. 5. Функции Ферми $F_{r+n}(\eta)$, где $n: 1,4-0.5,2,5-$ $1.5,3,6-2.5$ и зависимости коэффициента Зеебека от эффективного параметра рассеяния $r_{\mathrm{eff}},(7,8)$ для пленки $1,2,3,7$ и объемного твердого раствора $n$ - $\mathrm{Bi}_{2} \mathrm{Te}_{2.7} \mathrm{Se}_{0.15} \mathrm{~S}_{0.15}$ $4,5,6,8$.

$n-\mathrm{Bi}_{2} \mathrm{Te}_{2.7} \mathrm{Se}_{0.15} \mathrm{~S}_{0.15}$, чем в объемном образце во всем исследованном интервале температур (рис. 6, кривые 1,2), что определяется величинами параметра $r_{\text {eff }}$, который выше в пленке, чем в объемном материале, как показано на рис. 5 , кривые 7,8 .

С ростом температуры от 80 до $120-130 \mathrm{~K}$ величина $m / m_{0}$ в пленке заметно уменьшается, что связано с рассеянием на антиструктурных точечных дефектах. Однако при дальнейшем повышении температуры увеличивается влияние рассеяния на примесных дефектах замещения, $m / m_{0}$ возрастает и достигает максимума при температуре, которой соответствует максимальная величина термоэлектрической эффективности $Z(T)$ (рис. 3, кривые 3,4$)[28,38]$. Подвижность $\mu_{0}$, как и электропроводность $\sigma$, в пленке $n-\mathrm{Bi}_{2} \mathrm{Te}_{2.7} \mathrm{Se}_{0.15} \mathrm{~S}_{0.15}$ ниже, чем в объемном термоэлектрике (рис. 4, 6, кривые 3,4), что обусловлено компенсацией вкладов в проводимость акцепторных и донорных собственных дефектов [34-36].

Число Лоренца (рис. 7, врезка) при расчете электронной теплопроводности $\kappa_{e}=L \sigma T$ было определено в соответствии с (3) для значений $r_{\text {eff, }}$ приведенных на рис. 5 , кривые 7,8 .

$L=\left(\frac{k}{e}\right)^{2}\left(\frac{(r+7 / 2) F_{r+5 / 2}(\eta)}{(r+3 / 2) F_{r+1 / 2}(\eta)}-\frac{(r+5 / 2) F_{r+3 / 2}^{2}(\eta)}{(r+3 / 2) F_{r+1 / 2}^{2}(\eta)}\right)$.

Снижение величины $\kappa_{L}$ в пленке $n-\mathrm{Bi}_{2} \mathrm{Te}_{2.7} \mathrm{Se}_{0.15} \mathrm{~S}_{0.15}$ по сравнению с объемным материалом (рис. 7, кривые 1,2 ) объясняется сильной энергетической зависимостью длины свободного пробега фононов в узком интервале энергий, который существенно меньше, чем интервал энергий электронов [9,22]. При низких температурах уменьшение решеточной теплопроводности 


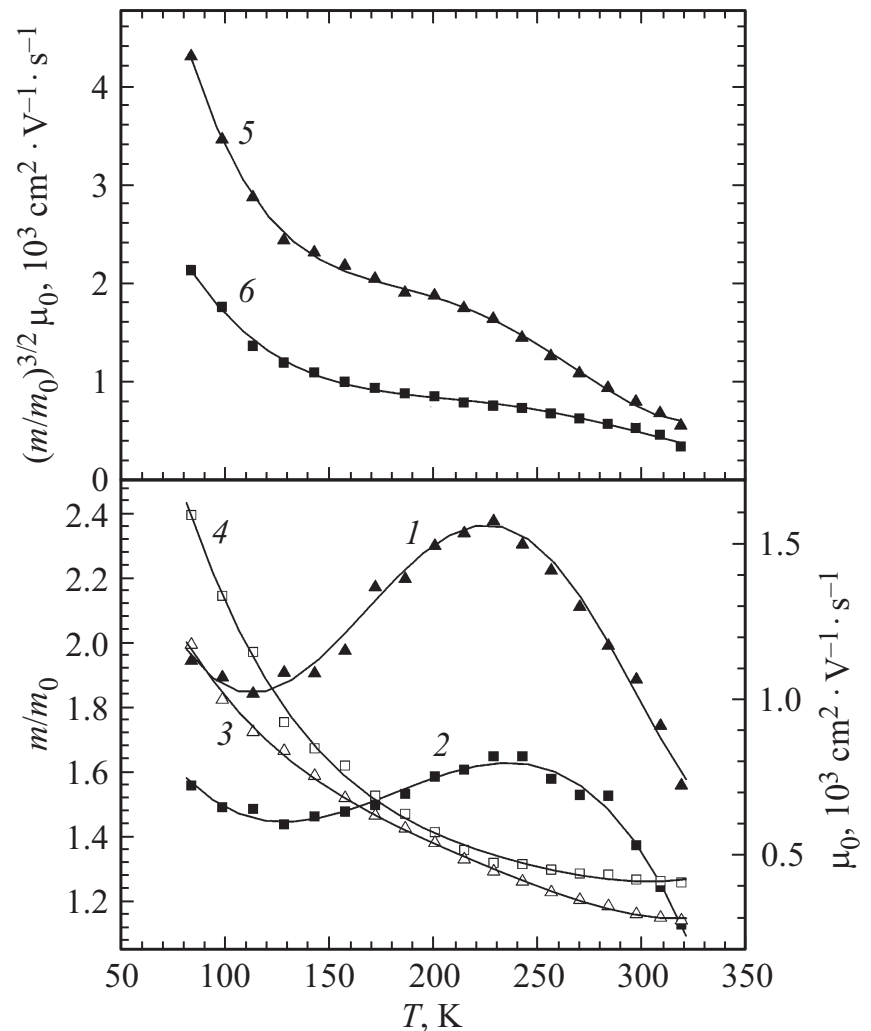

Рис. 6. Температурные зависимости эффективной массы плотности состояний $m / m_{0}(1,2)$, подвижности носителей заряда $\mu_{0}(3,4)$ и параметра материала $\left(m / m_{0}\right)^{3 / 2} \mu_{0}(5,6)$ в слоистой пленке $(1,3,5)$ и объемном твердом растворе $(2,4,6)$ $n-\mathrm{Bi}_{2} \mathrm{Te}_{2.7} \mathrm{Se}_{0.15} \mathrm{~S}_{0.15}$.

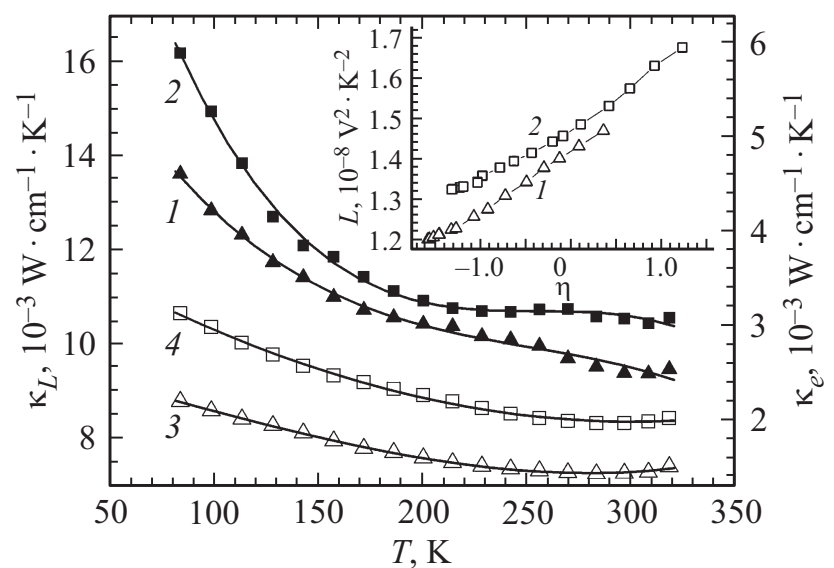

Рис. 7. Температурные зависимости теплопроводности кристаллической решетки $\kappa_{L}(1,2)$, электронной теплопроводности $\kappa_{e}(3,4)$ и числа Лоренца $L$ (вставка) в слоистой пленке $(1,3)$ и объемном твердом растворе $(2,4)$ $n-\mathrm{Bi}_{2} \mathrm{Te}_{2.7} \mathrm{Se}_{0.15} \mathrm{~S}_{0.15}$.

определяется в основном рассеянием на собственных и примесных точечных дефектах вплоть до температуры Дебая [32]. С повышением температуры влияние рассеяния на величину $\kappa_{L}$ на точечных дефектах снижается и преобладает рассеянием фононов на межкристаллитных границах в пленках топологических изоляторов $n-\mathrm{Bi}_{2} \mathrm{Te}_{2.7} \mathrm{Se}_{0.15} \mathrm{~S}_{0.15}$, вблизи комнатной температуры основным становится фонон-фононное рассеяние [28,38]. Снижение электронной теплопроводности $\kappa_{e}$ (рис. 7, кривые 3,4) в исследованных пленках определяется уменьшением электропроводности и подвижности. Таким образом, полученное снижение полной теплопроводности и значительный рост эффективной массы плотности состояния при увеличении эффективного параметра рассеяния, определяется усилением энергетической зависимости времени релаксации, что объясняют рост термоэлектрической эффективности в пленке топологического изолятора $n$ - $\mathrm{Bi}_{2} \mathrm{Te}_{2.7} \mathrm{Se}_{0.15} \mathrm{~S}_{0.15}$.

\section{5. Заключение}

Проведены исследования морфологии межслоевой поверхности (0001) методом сканирующей туннельной микроскопии и термоэлектрических свойств в слоистых пленках топологических изоляторов $n-\mathrm{Bi}_{2} \mathrm{Te}_{2.7} \mathrm{Se}_{0.15} \mathrm{~S}_{0.15}$ с оптимальными свойствами для температур ниже комнатной при высокой величине коэффициента Зеебека $S=-150$ и $-260 \mu \mathrm{VK}^{-1}$ при 80 и $290 \mathrm{~K}$ соответственно. На профилях поверхности (0001) обнаружены нейтральные примесные дефекты замещения $\mathrm{Se} \rightarrow \mathrm{Te}$ и $\mathrm{S} \rightarrow$ Те и собственные донорные антиструктурные дефек-

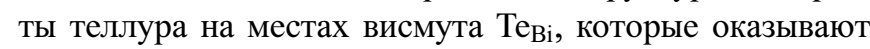
влияние на электронный и фононный транспорт в слоистых пленках.

Показано, что параметр мощности $S^{2} \sigma$ в пленах возрастает, поскольку рост коэффициента Зеебека компенсирует снижение электропроводности, что вместе с уменьшением теплопроводности обеспечивают рост средней величины термоэлектрической эффективности $\langle Z\rangle$ до $3.0 \cdot 10^{-3} \mathrm{~K}^{-1}$ в интервале температур 80-215 K. Получено, что усиление энергетической зависимости времени релаксации, характерное для топологических изоляторов, определяется ростом эффективного параметра рассеяния $r_{\text {eff }}$ с учетом которого были определены эффективная масса плотности состояний $m / m_{0}$, подвижность $\mu_{0}$, решеточная $\kappa_{L}$ и электронная теплопроводность $\kappa_{e}$. Значительный рост эффективной массы, определяющий увеличение параметра материала $\left(m / m_{0}\right) 3 / 2 \mu_{0}$, пропорционального фактору мощности $S^{2} \sigma$, и уменьшение величин $\kappa_{L}$ и $\kappa_{e}$ объясняют рост термоэлектрической эффективности в пленках топологических изоляторов $n-\mathrm{Bi}_{2} \mathrm{Te}_{2.7} \mathrm{Se}_{0.15} \mathrm{~S}_{0.15}$.

\section{Финансирование работы}

Финансовая поддержка исследований получена в рамках Российского фонда фундаментальных исследований, проект № 20-08-00464. 


\section{Конфликт интересов}

Авторы заявляют, что у них нет конфликта интересов.

\section{Список литературы}

[1] D.M. Rowe. Thermoelectric harvesting of low-temperature heat. In: Modules, Systems, and Applications in Thermoelectrics / Ed. D.M. Rowe. CRC Press, Boca Raton, FL (2012) P. 23-1.

[2] M.Z. Hasan, C.L. Kane. Rev. Mod. Phys. 82, 3045 (2010).

[3] С.И. Веденеев. УФН 187, 4, 411 (2017).

[4] N. Xu, Y. Xu, J. Zhu. npj Quant. Mater. 2, 51 (2017).

[5] G. Jiang, J. Yi, L. Miao, P. Tang, H. Huang, C. Zhao, S. Wen. Sci. Rep. 8, 2355 (2018).

[6] L. Zhang, J. Liu, J. Li, Z. Wang, Y. Wang, Y. Ge, W. Dong, Xu, H. Zhang, W. Zhang. Laser Photon. Rev. 14, 4, 1900409 (2020).

[7] W. Han, Y. Otani, S. Maekawa. npj Quantum Mater. 3, 27 (2018).

[8] R. Sun, S. Yang, X. Yang, E. Vetter, D. Sun, N. Li, L. Su, Yan Li, Yang Li, Z. Gong, Z. Xie, K. Hou, Q. Gul, W. He, X. Zhang, Z. Cheng. Nano Lett. 19, 4420 (2019).

[9] R.V. Gorbachev, A.K. Geim, M.I. Katsnelson, K.S. Novoselov, T. Tudorovskiy, I.V. Grigorieva, A.H. Mac Donald, S.V. Morozov, K. Watanabe, T. Taniguchi, L.A. Ponomarenko. Nature Phys. 8, 896 (2012).

[10] Y. Hou, R. Wang, R. Xiao, L. McClintock, H.C. Travaglini, J.P. Francia, H. Fetsch, O. Erten, S.Y. Savrasov, B. Wang, A. Rossi, I. Vishik, E. Rotenberg, D. Yu. Nature Comms. 10, 5723 (2019).

[11] J. Heremans, R. Cava, N. Samarth. Nature Rev. Mater. 2, 17049 (2017).

[12] Liu T.-H., Zhou J., Li M. et al. Proc. Natl. Acad. Sci. 115, 879 (2018).

[13] D. Baldomir, D. Failde. Sci. Rep. 9, 6324 (2019).

[14] D.Y.H. Ho, I. Yudhistira, B.Y.-K. Hu. Comms. Phys. 1, 9, 41 (2018).

[15] J. Hu, A.F. Rigosi, D.B. Newell, Y.P. Chen. Phys. Rev. B 102, 235304 (2020)

[16] M.M. Fogler, L.V. Butov, K.S. Novoselov. Nature Comms. 5, 4555 (2014).

[17] Y. Chen, Y. Huang, W. Lou, Y. Cai, K. Chang. Phys. Rev. B 102, 165413 (2020).

[18] B. Seradjeh, J.E. Moore, M. Franz. Phys. Rev. Lett. 103, 066402 (2009)

[19] K. Wu, L. Rademaker, J. Zaanen. Phys. Rev. Appl. 2, 054013 (2014).

[20] I.V. Korobeinikov, N.V. Morozova, L.N. Lukyanova, O.A. Usov, V.A. Kulbachinskii, V.V. Shchennikov, S.V. Ovsyannikov. J. Phys. D 51, 025501 (2018).

[21] И.В. Коробейников, Н.В. Морозова, Л.Н. Лукьянова, О.А. Усов, С.В. Овсянников. ФТП 53, 741 (2019).

[22] M.K. Jacobsen, R.S. Kumar, A.L. Cornelius, S.V. Sinogeiken, M.F. Nico. J. Phys.: Conf. Ser. 73, 9, 1154 (2012).

[23] M. Zhang, X. Wang, A. Rahman, Q. Zeng, D. Huang, R. Dai, Z. Wang, Z. Zhang. Appl. Phys. Lett. 112, 041907 (2018).

[24] Л.Н. Лукьянова, А.Ю. Бибик, В.А. Асеев, О.А. Усов, И.В. Макаренко, В.Н. Петров, Н.В. Никоноров. ФТП 51, 6 (2017).
[25] L.N. Lukyanova, I.V. Makarenko, O.A. Usov. J. Phys.: Condens. Matter 32, 465701 (2020).

[26] S. Jia, H. Beidenkopf, I. Drozdov, M.K. Fuccillo, J. Seo, J. Xiong, N.P. Ong, A. Yazdani. Phys. Rev. B 86, 165119 (2012).

[27] H. Beidenkopf, P. Roushan, J. Seo, L. Gorman, I. Drozdov, Y.S. Hor, R.J. Cava, A. Yazdani. Nature Phys. 7, 939 (2011).

[28] T. Zhu, L. Hu, X. Zhao, J. He. Adv. Sci. 3, 7, 1600004 (2016).

[29] A.M. Netsou, D.A. Muzychenko, H. Dausy, T. Chen, F. Song, K. Schouteden, M.J. Van Bael, C. Van Haesendonck. ACS Nano 14, 10, 13172 (2020).

[30] A. Hashibon, C. Elsasser. Phys. Rev. B 84, 144117 (2011).

[31] H. Nam, Y. Xu, I. Miotkowski, J. Tian, Y.P. Chen, C. Liu, C.K. Shih. J. Phys. Chem. Solids 128, 251 (2019).

[32] D. Bessas, I. Sergueev, H.-C. Wille, J. Person, D. Ebling, R.P. Hermann. Phys. Rev. B 86, 224301 (2012).

[33] J.C. Slater. J. Chem. Phys. 41, 3199 (1964).

[34] Z. Ren, A.A.Taskin, S. Sasaki, K. Segawa, Y. Ando. Phys. Rev. B. 84, 165311 (2011).

[35] A.A. Taskin, Z. Ren, S. Sasaki, K. Segawa, Y. Ando. Phys. Rev. Lett. 107, 016801 (2011).

[36] Y. Ando. J. Phys. Soc. Jpn. 82, 102001 (2013).

[37] L.N. Lukyanova, Yu.A. Boikov, Danilov, O.A. Usov, M.P. Volkov, V.A. Kutasov. Semicond. Sci. Technol. 30, 015011 (2015).

[38] P. Wei, J. Yang, L. Guo, S. Wang, L. Wu, X. Xu, W. Zhao, Q. Zhang, W. Zhang, M.S. Dresselhaus, J. Yang. Adv. Funct. Mater. 26, 29, 5360 (2016).

Редактор К.В. Емцев 